\title{
Relación entre los Problemas Emocionales y el Desarrollo del Lenguaje en Niños y Adolescentes con Dificultades Auditivas
}

\author{
The Relationship Between Emotional Problems and Language \\ Development in Children and Adolescents with Hearing Loss
}

\author{
Olga María Alegre ${ }^{1 *}$ \\ Luis Miguel Villar ${ }^{2}$ \\ ${ }^{1}$ Universidad de La Laguna, España \\ ${ }^{2}$ Universidad de Sevilla, España
}

\begin{abstract}
El presente estudio buscó conocer aspectos emocionales de niños y niñas con dificultades auditivas y cotejar su influencia en el desarrollo del lenguaje desde la perspectiva de los propios niños y niñas, sus padres y sus profesores de las islas de Gran Canaria y Tenerife. Se analizó el Cuestionario de Fortalezas y Dificultades (SDQ) administrado a 300 niños que empleaban implantes cocleares o audífonos comparado con las percepciones de sus familias y profesorado en el mismo cuestionario. Se aplicó un análisis de regresión para conocer si los resultados en el SDQ influyen en los obtenidos por los niños en el Test Illinois de Aptitudes Psicolingüísticas (ITPA). Los hallazgos mostraron que la valoración de las dimensiones emocionales de los niños y niñas en términos de fortalezas y debilidades, fueron juzgadas de manera diferente en los tres grupos de informantes estudiados, siendo la escala Problemas de conducta la cuestión emocional de mayor dificultad planteada. Se correlacionaron ambos instrumentos evaluativos mostrando el análisis de regresión que la puntuación vinculada a la medida de los aspectos emocionales predice los resultados en las diferentes dimensiones de ITPA. Los hallazgos de este estudio son pertinentes para el diseño de programas educativos en el ámbito de la comunicación y del lenguaje de niños y niñas.
\end{abstract}

Descriptores: Dificultad en el aprendizaje; Educación de sordos; Educación especial; Lenguaje hablado; Recepción auditiva.

\begin{abstract}
The present study sought to know emotional aspects of children with hearing difficulties and to check their influence on language development from the perspective of the children themselves, their parents and their teachers of Gran Canaria and Tenerife islands. We analyzed the Strengths and Difficulties Questionnaire (SDQ) administered to 300 children who used cochlear implants or hearing aids compared to the perceptions of their families and teachers in the same questionnaire. In addition, a regression analysis was applied to know if the results in the SDQ influence those obtained by the children in the Illinois Psycholinguistic Aptitude Test (ITPA). The findings showed that the assessment of the emotional dimensions of the children in terms of strengths and weaknesses were judged differently in the three groups of informants studied, the scale being the most difficult emotional issue. Both evaluative instruments were correlated and a linear regression analysis that the score linked to the measurement of the emotional aspects predicts the results in the different dimensions of ITPA. The findings of this study are relevant for the design of educational programs in the field of communication and language of children.
\end{abstract}

Keywords: Auditory reception; Difficulty in learning; Education of the deaf; Special needs education; Hearing.

*Contacto: oalegre@gmail.com

ISSN: 1696-4713

www.rinace.net/reice/

revistas.uam.es/reice
Recibido: 1 de noviembre 2018

$1^{\text {a }}$ Evaluación: 18 de noviembre 2018

$2^{\text {a }}$ Evaluación: 25 de noviembre 2018

Aceptado: 29 de noviembre 2018 


\section{Introducción}

Los aspectos emocionales, medidos como fortalezas y debilidades, no suelen vincularse a las aptitudes lingüísticas en niños y adolescentes con dificultades auditivas y, en concreto, a aquellos que emplean dispositivos auditivos, como son implantes cocleares (IC) o audífonos (AUD).

Sin embargo, es conocido que casi la mitad de los problemas emocionales y mentales se inician antes de los 14 años y que una buena salud mental es prerrequisito para el desarrollo óptimo de la infancia. La evaluación de las funciones psicolingüísticas implicada en el proceso de comunicación tiene especial relieve en la infancia. Justamente, las personas con dificultades auditivas tienen interferencias en la comunicación que afectan sus percepciones y conocimientos, que resultan fundamentales para detectar problemas de aprendizaje. Calderón y Greenberg (2003) sostienen que el funcionamiento socioemocional competente es el resultado de la capacidad para coordinar afecto, cognición, comunicación y comportamiento y destacan que los retrasos en el desarrollo lingüístico y la falta de experiencias sociales mediadas por el lenguaje, generan brechas importantes en el desarrollo emocional.

Este estudio tiene como objetivo principal conocer las autopercepciones de fortalezas y dificultades emocionales de niños y adolescentes que emplean dispositivos auditivos (IC o AUD) y comparar dichas apreciaciones con las percepciones de sus padres y madres, así como las percepciones de su profesorado. Asimismo, analizar las aptitudes psicolingüísticas de niños y adolescentes e investigar los problemas emocionales de niños y adolescentes como predictores de sus aptitudes comunicativas y de lenguaje, con objeto de diseñar programas educativos basados en aptitudes psicolingüísticas, cursos de formación para padres y madres, y talleres de desarrollo profesional docente de educación inclusiva para estudiantes con dificultades auditivas.

La presente investigación ha contado con una amplia muestra de niños y adolescentes con dificultades auditivas, así como de sus padres y madres y maestros (tutores y especialistas en audición y lenguaje) de centros escolares públicos de las islas de Gran Canaria y Tenerife.

\section{Revisión de la literatura}

El estudio del lenguaje en niños y adolescentes con dificultades auditivas ha sido una constante en la investigación educativa y es conocido que el lenguaje oral no se adquiere de forma natural cuando se tienen tales dificultades, sino que es preciso tener una educación proyectada. Este proceso está afectado por la influencia de variados factores como son las emociones.

La Organización Mundial de la Salud (2005) señaló que una buena salud mental es prerrequisito para el desarrollo psicológico óptimo de la infancia siendo la prevención una de las estrategias más importantes a implantar. En la actualidad existen diferentes métodos estandarizados de medición que permiten realizar diagnósticos tempranos relacionados con los problemas emocionales y psicológicos infantiles. 


\subsection{Características de los problemas de salud mental en niños y adolescentes con dificultades auditivas}

El Cuestionario de Capacidades y Dificultades (SDQ), diseñado por Goodman (2001), fue concebido como un instrumento para realizar el cribado del comportamiento de socialización y psicopatología de niños de 3 a 16 años. Ha sido traducido a más de 60 idiomas. Los investigadores Sharp y otros (2005) argumentaron el valor predictivo de SDQ utilizando muestras de una comunidad de niños ingleses estableciendo que los informes de los maestros tuvieron mayor capacidad predictiva de la conducta emocional de los niños que los padres. Precisamente, la conducta estresada de los padres y su relación con el desarrollo socioemotivo de los niños fue objeto de un análisis multivariante en la investigación de Hintermair (2006).

Goodman y Goodman (2009), apoyaron el uso del SDQ como medida de la salud mental infantil, que completaron padres, maestros y niños o adolescentes de 5 a 16 años. Otro ejemplo ha sido el estudio de Gustafsson, Proczkowska-Björklund y Gustafsson (2017) que aplicaron el SDQ en la versión de maestros a niños de 1-5 años en Suecia. RodríguezHernández y otros (2014) concluyeron en un estudio español que los puntos de corte de las escalas de SDQ obtenidos eran distintos que los conseguidos en otros países. Posteriormente, Ortuño-Sierra y colaboradores (2016) evidenciaron las propiedades psicométricas de SDQ en España.

La investigación con niños y adolescentes con dificultades auditivas no ha sido ajena a la utilización de herramientas metodológicas para conocer las dificultades psicoemotivas de los mismos. Desde este ángulo, Dammeyer (2010) evaluó una población danesa que tenía dificultades o pérdida de audición entre un 20\% a 50\%, subrayando la significación expresada por otros autores acerca de la comunicación en el desarrollo psicológico de niños con dificultades auditivas.

Bajo la idea de que era importante tratar los problemas mentales, Huber y Kipman (2011) utilizaron SDQ para evaluar la condición mental de 32 adolescentes con implante coclear (edad media de 15.0 años) y de 212 coetáneos con audición típica (edad media de 15.0 años) en Austria. Como conclusión declararon:

\footnotetext{
Los hallazgos indicaron que la salud mental de los adolescentes sordos con implante coclear era comparable a la de sus coetáneos con audición normal, aunque los maestros clasificaron a los que tenían implante coclear con más problemas con compañeros y problemas "totales". (p. 152)
}

En el estudio comparativo entre las autopercepciones de niños y adolescentes, y las percepciones de padres y profesores sobre las fortalezas y debilidades de niños y adolescentes con implante coclear, Anmyr y otros (2012) concluyeron que "los niños con implante coclear no mostraron más dificultades o problemas de salud mental que los niños y niñas con audición típica, aunque sus padres dieron cuenta de más fortalezas de las que los niños hicieron" (p. 1111).

Dentro de los problemas de salud mental de niños y adolescentes con dificultades auditivas, Hintermair (2013) estudió la función ejecutiva y la competencia comunicativa de escolares que fueron evaluados por su profesorado. De nuevo, el SDQ se ha utilizado para validar la estructura del instrumento en Dinamarca. Utilizando dos muestras diferentes en dos periodos temporales distintos (2007 y 2014), Niclasen y Dammeyer (2016, p. 134) utilizaron dos análisis factoriales (uno exploratorio y otro confirmatorio) averiguando que en las dos muestras se mantenía la estructura de los cinco factores: 
hiperactividad, desorden de conducta, problemas emocionales, problemas con los compañeros y destrezas prosociales, independientemente de que los informantes en la muestra de niños y adolescentes de 2007 fuera el profesorado y los padres y madres en la muestra de 2014.

\subsection{Habilidades psicolingüísticas de niños con dificultades auditivas}

Ante la presencia de dificultades auditivas en niños y adolescentes, su capacidad lingüística puede verse afectada perjudicando elementos que intervienen en la comunicación, como vocabulario, gramática, articulación, fluidez, comprensión o pronunciación, entre otros. La adquisición del lenguaje oral no es un proceso espontáneo y natural, vivido en situaciones cotidianas de comunicación e intercambio socio-familiar, sino que supone un aprendizaje planificado de manera sistemática.

Menos usado que el cuestionario SDQ aplicado a la población infantil con dificultades auditivas ha sido la exploración de los problemas de lenguaje de niños y adolescentes por medio del ITPA. Test Illinois de Aptitudes Psicolingüísticas (Bateman, 1965; Kirk y Elkins, 1974). Aplicado este test a niños y adolescentes con dificultades auditivas, se ha sondeado cómo se relacionaban las áreas de vulnerabilidad de niños y adolescentes con sus diferencias individuales en la comprensión lectora. Los niños y adolescentes con implante coclear desarrollan habilidades de lenguaje casi dentro de parámetros típicos. Sin embargo, existe una gran variación en los resultados individuales después de la implantación coclear o del uso de audífonos.

El ITPA se ha usado, además, para establecer la validez concurrente y predictiva de otros instrumentos de diagnóstico del deterioro específico del lenguaje (Girbau, 2016) o para comprobar su potencia predictiva. Así, comparados el test ITPA y la Escala de inteligencia de Wechsler para niños revisada (WISC-R), ambos complementarios para evaluar niños y adolescentes con dificultades de lenguaje, Ottem (2010) concluyó que el ITPA era más sensible para captar las diferencias entre las habilidades verbales y el rendimiento de niños y adolescentes que el WISC-R.

Otros trabajos de investigación se han centrado en el deterioro específico del lenguaje. Así, Girbau (2016) aplicó tres subtests del ITPA (comprensión auditiva, asociación auditiva e integración gramatical), entre otras pruebas, para determinar el uso potencial de la versión española de la prueba "Tarea de repetición sin palabras" como un marcador que diagnosticaba a niños y adolescentes en el deterioro específico del lenguaje. También se ha diagnosticado el desorden del espectro de autismo usando el ITPA para validar las escalas cognitivas y del lenguaje del test Bayley-III (Torras-Mañá et al., 2016).

El estudio de Hassan, Eeldin y Al Kasaby (2014) consistió en averiguar qué habilidades psicolingüísticas tenían niños y adolescentes de habla árabe con pérdida auditiva neurosensorial congénita o con implante coclear en comparación con niños y adolescentes sin dificultades auditivas aplicando el ITPA, obteniendo como resultado que no había diferencias estadísticamente significativas entre los tres grupos.

\subsection{Metas e hipótesis del estudio}

El presente estudio tuvo cuatro metas orientadas a investigar el objetivo general relativo a cómo influyen los problemas y dificultades emocionales en el desarrollo del lenguaje de niños y adolescentes con dificultades auditivas. Similares propuestas investigadoras 
habían realizado otros autores (Anmyr et al., 2012; Bat-Chava, Martín y Kosciw, 2005; Hintermair, 2013):

- Analizar y comparar la igualdad o diferencia entre las autopercepciones de niños y adolescentes con IC o AUD y las percepciones de sus familias (padres o madres) y maestros (especialistas y tutores) de las fortalezas y dificultades emocionales de dichos niños y adolescentes, medidas por las subescalas de SDQ (meta 1).

- Investigar y comparar las diferencias entre las características socioeducativas de los niños y adolescentes con dificultades auditivas en cada uno de los subtests del ITPA (meta 2).

- Correlacionar las puntuaciones de las subescalas de SDQ y los niveles del ITPA en niños y adolescentes con IC o AUD (meta 3).

- Establecer un modelo de predicción de puntuaciones de ITPA en niños y adolescentes con IC o AUD a partir de la puntuación total de SDQ (meta 4).

Las hipótesis quedaron formuladas como sigue:

- En relación con la meta 1, se rechaza la hipótesis nula de la concordancia entre las medias de los distintos sujetos del estudio.

- Vinculada a la meta 2, se rechaza la hipótesis nula de la concomitancia entre las medias de las distintas variables socioeducativas.

- Respecto de la meta 3, se expresó la existencia de correlaciones entre las subescalas de SDQ y los niveles de ITPA.

- Perteneciente a la meta 4, se declaró que la puntuación final de SDQ predecía las puntuaciones de ITPA.

\section{Método}

\section{Participantes}

La muestra de niños y adolescentes estudiada con dificultades auditivas fue la siguiente: 187(62,3\%) niños y adolescentes con IC y $113(37,7 \%)$ que usaban AUD, que procedían de las dos provincias de las Islas Canarias: $163(54,3 \%)$ estudiantes acudían a centros escolares de Las Palmas de Gran Canaria y 137 (45,7\%) de Santa Cruz de Tenerife. Fueron seleccionados de entre los niños y adolescentes con dificultades auditivas de Canarias que usaban IC o AUD y no tenían otros déficits asociados, a juicio del Equipo especialista de atención al alumnado con dificultades auditivas del Gobierno de Canarias. En el estudio había más niños y adolescentes $(\mathrm{n}=163)$ que niñas y adolescentes $(\mathrm{n}=137)$. Los escolares cursaban estudios en Educación Primaria $(n=185)$, Secundaria Obligatoria $(n=104), y$ Bachillerato/Formación Profesional $(n=11)$. Los niños y adolescentes que no tenían Adaptaciones Curriculares Individualizadas (ACIs) en su enseñanza eran más numerosos $(\mathrm{n}=181)$ que los que sí las tenían $(\mathrm{n}=119)$. Habían recibido una calificación promedio en el curso anterior de Suficiente $(n=107)$, seguida de Bien $(n=79)$, Insuficiente $(n=56)$, Notable $(\mathrm{n}=47)$, y Sobresaliente $(\mathrm{n}=11)$. Respecto al número de horas semanales que los niños y adolescentes asistían a sesiones de logopedia extraescolares, 89(29,7\%) indicaron que acudían a clase una hora a la semana; $68(22,7 \%)$ frecuentaban las clases dos horas a la semana; $66(22,0 \%)$ estaban tres horas semanales, y $30(10,0 \%)$ acudían a las sesiones de logopedia cuatro horas a la semana. En cuanto a la muestra del profesorado 
tutor $(\mathrm{n}=300)$ un total de 185 tenían 10 o más años de experiencia docente; entre 7-9 años de experiencia docente $(n=42)$; entre 4-6 años $(n=32)$ y con 3 o menos años de experiencia $(n=41)$. Asimismo, la experiencia docente de la muestra de profesorado especialista en audición y lenguaje $(\mathrm{n}=300)$ era de 10 o más años de experiencia docente $(\mathrm{n}=193)$, seguida de aquellos con 7-9 años de experiencia docente $(\mathrm{n}=89$, con 4-6 años de experiencia docente $(n=10)$, y los que tenían 3 o menos años de experiencia $(n=8)$. Las familias constituyeron la última muestra de participantes $(\mathrm{n}=300$ : padres $\mathrm{n}=37$, madres $n=242$, ambos $n=2$, tutor $n=17$, hermano $n=2$ ). Las madres tenían la siguiente educación formal: Educación Primaria $(\mathrm{n}=89)$, Educación Superior $(\mathrm{n}=87)$, Bachillerato $(\mathrm{n}=70)$, sin estudios $(\mathrm{n}=37)$ y Formación Profesional $(\mathrm{n}=17)$. Mientras que la educación de los padres fue: Educación Primaria $(\mathrm{n}=87)$, sin estudios $(\mathrm{n}=73)$, Educación Superior $(\mathrm{n}=65)$, Bachillerato $(\mathrm{n}=53)$ y Formación Profesional $(\mathrm{n}=22)$. Finalmente, la comunicación de los estudiantes con sus familias era mayoritariamente oral $(\mathrm{n}=215)$, bilingüe $(\mathrm{n}=57)$ o por lengua de signos $(\mathrm{n}=28)$. La población de niños y adolescentes con IC o AUD del estudio fue proporcionada por la Consejería de Educación y Universidades del Gobierno de Canarias. Asimismo, la institución canaria puso a disposición de los investigadores el archivo de los centros escolares públicos. Los datos personales, sociales y académicos de la muestra de niños y adolescentes con IC o AUD se utilizaron como variables independientes en la meta 2.

\section{Instrumentos}

Se utilizaron dos instrumentos para determinar las fortalezas y dificultades emocionales y conductuales, y las habilidades psicolingüísticas de los niños y adolescentes:

- SDQ. Cuestionario de fortalezas y dificultades, versiones para niños, padres y maestros: Los 25 ítems se dividen en cinco subescalas (cinco ítems cada una) que incluyen síntomas emocionales, problemas de conducta, hiperactividad-falta de atención, problemas de relación entre iguales y conducta prosocial. Los ítems se pueden sumar para generar un puntaje total de dificultades (20 ítems). En nuestro estudio se obtuvo un valor 0,856 en el alfa de Cronbach para SDQ, similar a los valores obtenidos en los estudios de Goodman (2001) y Ruchkin y otros (2012).

- ITPA. Test Illinois de Aptitudes Psicolingüísticas: El test postula tres dimensiones de habilidades psicolingüísticas: dos niveles de organización, cuatro canales de comunicación y tres procesos. Los seis subtests del nivel de representación requieren comprender y dar significado a los símbolos de la comunicación. Dos tipos de subtests evalúan el nivel secuencial automático definidos en términos del canal. En este estudio se obtuvo un valor 0,771 en el alfa de Cronbach para ITPA, similar a los obtenidos por Weener, Barritt y Semmel (1967) y Kirk y Elkins (1974).

\section{Procedimiento}

Los niños y adolescentes que usaban un IC como dispositivo auditivo cumplimentaron las pruebas administradas por logopedas entrenados en la Unidad de Hipoacusia del Hospital de referencia, al que acudían regularmente para las programaciones del IC (todos implantados antes de los 3 años de edad), mientras que los niños y adolescentes con AUD los cumplimentaron en horas de tutoría en sus centros educativos administrados por logopedas entrenados. Los padres y madres de los niños y adolescentes autorizaron por 
escrito la participación en el estudio de sus hijos e hijas y respondieron al cuestionario SDQ en sus hogares. Debían hacerlo en el plazo de dos semanas máximo. Por su parte, el profesorado respondió al cuestionario SDQ en los centros educativos, en hora de tutoría. De manera acordada con los maestros, los niños cumplimentaron el ITPA en otra sesión de tutoría para evitar la fatiga de los niños y adolescentes. El proceso de contacto inicial con los centros educativos, exposición de los objetivos del estudio y recogida de datos cumplimentados de SQD e ITPA duró aproximadamente un año escolar. La dirección de los centros educativos públicos ejerció un liderazgo inclusivo abriéndose a la investigación para la participación y mejora de la comunidade educativa (León, Crisol y Moreno, 2018).

\section{Análisis de datos}

Mediante un enfoque cuantitativo de carácter empírico y un método hipotético-deductivo, se analizaron los datos aportados por los cuestionarios, usando el Statistical Package for Social Sciences (v. 27). Para la meta 1, se utilizó un Análisis Multivariado de la Varianza (MANOVA) para comparar los niños y adolescentes que usaban ICS con los que utilizaban AUDS, sus padres y profesores (especialistas y tutores) en las subescalas del SQD. Para la meta 2 se utilizó un MANOVA para cotejar las variables socioeducativas planteadas en la descripción de la muestra de este estudio con los resultados de los niños y adolescentes con IC o AUD en los subtests de ITPA. La meta 3 se analizó mediante correlaciones entre las subescalas del SDQ y los niveles del test ITPA para los niños y adolescentes con IC o AUD. Finalmente, la meta 4 empleó un análisis de regresión lineal utilizando como predictor la puntuación final de SDQ obtenida por los niños y adolescentes con IC o AUD y los resultados de los mismos en el ITPA.

\section{Resultados}

\subsection{La autopercepción de niños y adolescentes, y las percepciones de sus familias y maestros sobre sus fortalezas y dificultades emocionales}

Esta primera sección de resultados busca dar respuesta al primero de los objetivos del estudio: Analizar y comparar la igualdad o diferencia entre las autopercepciones de niños y adolescentes con IC o AUD las percepciones de sus familias (padres o madres) y maestros (especialistas y tutores) de las fortalezas y dificultades emocionales de los estudiantes medidas por las subescalas de SDQ. Al ser la muestra mayor que 100 se aplicó el test Kolmogorov-Smirnov para conocer su distribución normal. Se aplicó la prueba de Box sobre la igualdad de las matrices de covarianzas. También existió homocedasticidad entre las varianzas de los grupos, según la prueba de Levene para valores superiores a 0,05. Los resultados de la meta 1 se muestran en el cuadro 1.

En el caso de los niños y adolescentes, el valor $\lambda$ de Wilks $(0,48)$ en la comparación IC/AUD fue $p<0,001$. El $\eta^{2}$ parcial, explicó el modelo para el 98\% de los casos. El ANOVA reveló unas medias más elevadas en el caso de los niños y adolescentes que emplean el IC para todas las escalas excepto en "Síntomas emocionales y Prosocial" cuyas medias son superiores en el caso de aquellos que usan AUD. Las familias consiguieron resultados en la misma dirección para todas las escalas con superioridad en las medias al referirse a sus hijos con IC, excepto al evaluar las escalas "Síntomas emocionales y Prosocial" que es superada por la valoración de los padres de niños y adolescentes con AUD. El $\eta^{2}$ parcial emitió una explicación del modelo en el $86 \%$ de los casos $(\lambda=0,914, p<0,001)$. 
Cuadro 1. Resultados del MANOVA en las subescalas del SDQ referidos a los niños y adolescentes con IC o AUD, familias y maestros

\begin{tabular}{|c|c|c|c|}
\hline SUBESCALAS & $t$ & $\begin{array}{c}\text { IC } \\
\bar{x}(\sigma) \\
\end{array}$ & $\begin{array}{l}\text { AUD } \\
\bar{x}(\sigma)\end{array}$ \\
\hline \multicolumn{4}{|l|}{ Niños y niñas: } \\
\hline \multicolumn{4}{|c|}{ MANOVA $\lambda=0,480 . F=63,770 \cdot p<0,001 \cdot \eta^{2}$ parcial $=0,098$} \\
\hline Síntomas emocionales & $-5,374^{* * *}$ & $2,42(0,788)$ & $2,85(0,427)$ \\
\hline Problemas conducta & $6,876^{* * * *}$ & $2,71(0,599)$ & $2,13(0,840)$ \\
\hline Hiperactividad & $10,226^{* * *}$ & $2,84(0,471)$ & $2,07(0,831)$ \\
\hline Problema con compañeros & $10,323^{* * * *}$ & $2,83(0,451)$ & $2,08(0,814)$ \\
\hline Prosocial & $-10,581^{* * * *}$ & $1,18(0,439)$ & $1,99(0,881)$ \\
\hline \multicolumn{4}{|l|}{ Familias de niños y niñas } \\
\hline \multicolumn{4}{|c|}{ MANOVA $\lambda=0,914 . F=6.942 . p<0,000 \cdot \eta^{2}$ parcial $=0,086$} \\
\hline Síntomas emocionales & & $2,18(0,903)$ & $2,26(0,753)$ \\
\hline Problemas conducta & $4,949^{* * *}$ & $2,84(0,545)$ & $2,45(0,813)$ \\
\hline Hiperactividad & $5,064^{* * * *}$ & $2,84(0,545)$ & $2,44(0,812)$ \\
\hline Problema con compañeros & $5,140^{* * * *}$ & $2,84(0,545)$ & $2,43(0,822)$ \\
\hline Prosocial & $-5,140^{* * * *}$ & $1,16(0,545)$ & $1,57(0,822)$ \\
\hline \multicolumn{4}{|l|}{ Profesorado especialista } \\
\hline \multicolumn{4}{|c|}{ MANOVA $\lambda=0,565 . F=32,090 \cdot p<0,000 \cdot \eta^{2}$ parcial $=0,045$} \\
\hline Síntomas emocionales & & $2,16(0,883)$ & $2,08(0,846)$ \\
\hline Problemas conducta & & $2,65(0,767)$ & $2,64(0,482)$ \\
\hline Hiperactividad & & $2,48(0,850)$ & $2,56(0,500)$ \\
\hline Problema con compañeros & $8,926^{* * * *}$ & $2,94(0,287)$ & $2,23(0,858)$ \\
\hline Prosocial & $-8,545^{* * *}$ & $1,08(0,294)$ & $1,75(0,846)$ \\
\hline \multicolumn{4}{|l|}{ Profesorado tutor } \\
\hline \multicolumn{4}{|c|}{ MANOVA $\lambda=0,503 \cdot F=58,058 \cdot p<0,000 \cdot \eta^{2}$ parcial $=0,049$} \\
\hline Síntomas emocionales & & $2,26(0,829)$ & $2,27(0,907)$ \\
\hline Problemas conducta & $10,892^{* * * *}$ & $2,62(0,719)$ & $1,67(0,749)$ \\
\hline Hiperactividad & $8,108^{* * * *}$ & $2,86(0,466)$ & $2,30(0,731)$ \\
\hline Problema con compañeros & $8,332^{* * * *}$ & $2,89(0,401)$ & $2,29(0,842)$ \\
\hline Prosocial & $-11,884^{* * * *}$ & $1,19(0,521)$ & $2,12(0,832)$ \\
\hline
\end{tabular}

Nota: ${ }^{*} p<0,05 ;{ }^{* *} p<0,010 ;{ }^{* * *} p<0,001$.

Fuente: Elaboración propia.

El profesorado especialista de niños y adolescentes con IC presentaron medias superiores al profesorado especialista de niños y adolescentes con AUD en las escalas Síntomas emocionales, Problemas de conducta y Problema con los compañeros, mientras que el profesorado de aquellos que empleaban los AUD reflejaron medias superiores a las obtenidas por el profesorado de niños y adolescentes con IC en las escalas: Hiperactividad y Prosocial. El valor $\lambda$ de Wilks reveló la presencia de diferencias en función del tipo de dispositivo auditivo de los niños y adolescentes: Wilkis $\lambda=0,565, p<0,001$. Los resultados del profesorado tutor $\left(\lambda=0,503, F=58,058, p<0,001, \eta^{2}\right.$ parcial $\left.=0,049\right)$ mostraron diferencias significativamente superiores en los puntajes de las escalas Problemas de conducta e Hiperactividad que el grupo de profesorado de niños y adolescentes con IC. 


\subsection{Diferencias entre las características socioeducativas de los niños y adolescentes}

Los resultados de la meta 2: "Investigar y comparar las diferencias entre las características socioeducativas de los niños y adolescentes con IC o AUD en cada uno de los subtests del ITPA" se han reflejado en los cuadros 2,3 y 4 .

Cuadro 2. Resultados del MANOVA por variables socioeducativas de niños y adolescentes con IC o AUD en el Subtest del ITPA Nivel Automático: Auditivo-Vocal

\begin{tabular}{|c|c|c|c|c|}
\hline VARIABLES & SUBTEST & MANOVA & $t / F$ & $\bar{x}$ \\
\hline $\begin{array}{l}\text { Dispositivo } \\
\text { Auditivo }\end{array}$ & $\begin{array}{c}\text { Memoria } \\
\text { visomotora }\end{array}$ & $\begin{array}{c}\lambda=0,854 \\
F=8,357^{* * *} \\
\eta^{2} \text { parcial }=0,146\end{array}$ & $t=-4,345^{* * *}$ & $\begin{array}{l}\mathrm{IC}(31,97) \\
\operatorname{AU}(38,76)\end{array}$ \\
\hline Provincia & $\begin{array}{l}\text { Memoria } \\
\text { visomotora }\end{array}$ & $\begin{array}{c}\lambda=0,773 \\
F=14,354^{* * *} \\
\eta^{2} \text { parcial }=0,227\end{array}$ & $t=-4,345^{* * *}$ & $\begin{array}{l}\mathrm{T}(31,97) \\
\mathrm{LP}(38,76)\end{array}$ \\
\hline \multirow[t]{2}{*}{ Calificación } & $\begin{array}{l}\text { Memoria } \\
\text { visomotora }\end{array}$ & \multirow{2}{*}{$\begin{array}{c}\lambda=0,788 \\
F=3,000^{* *} \\
\eta^{2} \text { parcial }=0,198\end{array}$} & $F=5,166^{* * * *}$ & $\begin{array}{c}\mathrm{I}(39,13), \mathrm{S}(31,10) \\
\mathrm{B}(36,50), \mathrm{N}(31,79) \\
\text { So }(40,22)\end{array}$ \\
\hline & $\begin{array}{l}\text { Integración } \\
\text { visual }\end{array}$ & & $F=2,798^{*}$ & $\begin{array}{c}\mathrm{I}(31,80), \mathrm{S}(39,32) \\
\mathrm{B}(35,51), \mathrm{N}(37,67) \\
\text { So }(41,56)\end{array}$ \\
\hline \multirow{2}{*}{$\begin{array}{l}\text { Horas de } \\
\text { logopedia }\end{array}$} & $\begin{array}{l}\text { Integración } \\
\text { gramatical }\end{array}$ & \multirow{2}{*}{$\begin{array}{c}\lambda=0,869 \\
F=1,737^{*} \\
\eta^{2} \text { parcial }=0,035\end{array}$} & $F=2,480^{*}$ & $\begin{array}{c}\mathrm{N}(26,00), 1 \mathrm{~h}(21,15) \\
2 \mathrm{~h}(24,22), 3 \mathrm{~h}(20,47) \\
4 \mathrm{~h}(15,72)\end{array}$ \\
\hline & $\begin{array}{l}\text { Integración } \\
\text { visual }\end{array}$ & & $F=3,248^{*}$ & $\begin{array}{c}\mathrm{N}(36,10), 1 \mathrm{~h}(38,11) \\
2 \mathrm{~h}(40,78), 3 \mathrm{~h}(35,04), \\
4 \mathrm{~h}(30,50)\end{array}$ \\
\hline \multirow{2}{*}{$\begin{array}{l}\text { Años de } \\
\text { experiencia } \\
\text { profesor tutor }\end{array}$} & $\begin{array}{c}\text { Integración } \\
\text { gramatical }\end{array}$ & \multirow{2}{*}{$\begin{array}{c}\lambda=0,813 \\
F=3,480^{* * *} \\
\eta^{2} \text { parcial }=0,067\end{array}$} & $F=8,044^{* * * *}$ & $\begin{array}{l}<3(16,57), 4-6(35,13) \\
7-9(28,75),>10(19,82)\end{array}$ \\
\hline & $\begin{array}{c}\text { Integración } \\
\text { visual }\end{array}$ & & $F=3,353^{*}$ & $\begin{array}{l}<3(37,77), 4-6(45,44) \\
7-9(36,84),>10(35,83)\end{array}$ \\
\hline $\begin{array}{l}\text { Estudios del } \\
\text { padre }\end{array}$ & $\begin{array}{l}\text { Memoria } \\
\text { auditiva }\end{array}$ & $\begin{array}{c}\lambda=0,871 \\
F=1,700^{*} \\
\eta^{2} \text { parcial }=0,034\end{array}$ & $F=3,069^{*}$ & $\begin{array}{c}\mathrm{Se}(20,65), \operatorname{Pr}(19,98) \\
\mathrm{Ba}(18,13), \mathrm{Su}(27,00), \\
\operatorname{Fp}(25,33)\end{array}$ \\
\hline \multirow{2}{*}{$\begin{array}{l}\text { Comunicación } \\
\text { en familia }\end{array}$} & $\begin{array}{c}\text { Memoria } \\
\text { visomotora }\end{array}$ & \multirow{2}{*}{$\begin{array}{c}\lambda=0,867 \\
F=3,617^{* * * *} \\
\eta^{2} \text { parcial }=0,069\end{array}$} & $F=3,688^{*}$ & $\begin{array}{c}\mathrm{S}(40,70), \mathrm{O}(33,60) \\
\mathrm{B}(34,00)\end{array}$ \\
\hline & $\begin{array}{c}\text { Integración } \\
\text { visual }\end{array}$ & & $F=10,504^{* * * *}$ & $\begin{array}{c}\mathrm{S}(28,30), \mathrm{O}(39,01) \\
\mathrm{B}(33,06)\end{array}$ \\
\hline
\end{tabular}

Nota: ${ }^{*} p<0,05 ;{ }^{* *} p<0,010 ;{ }^{* * *} p<0,001$. Dispositivo Auditivo: Implante coclear (IC), Audífono (AU). Provincia: Tenerife (TF), Las Palmas de GC (LP). Calificaciones: Insuficiente(I), Suficiente (S), Bien (B), Notable (N), Sobresaliente (So). Horas de logopedia: Ninguna (N), 1h, 2h, 3h, 4h. Años de experiencia profesor tutor: Menos de 3 años (<3), entre 4-6 años, entre 7-9 años, más de 10 años $(<10)$. Estudios del padre: Sin estudios (SE), Estudios Primarios (PR), Bachillerato (BA), Estudios Superiores (SU), Formación Profesional (FP). Comunicación en familia (tipo): Signos (S), Oral $(\mathrm{O})$, Bilingüe $(\mathrm{B})$.

Fuente: Elaboración propia.

Por ejemplo, puede observarse en el cuadro 2 que se hallaron diferencias estadísticamente significativas en el Nivel Automático Auditivo-Vocal para el subtest Memoria Visomotora $[F(8.357), p<0,001, \lambda$ de Wilks $=0,854$ y eta parcial cuadrado $=0,146]$, en función del 
tipo de dispositivo auditivo, con mejor resultado de media para niños y adolescentes con AUD. El mismo subtest obtiene diferencias claras en las Calificaciones obteniendo menores resultados en dicha Memoria Visomotora aquellos niños y adolescentes con mejores notas como son los que obtienen Sobresaliente $(\bar{x}=41,56)$.

Cuadro 3. Resultados del MANOVA por variables socioeducativas de niños y adolescentes con IC o AUD en el Subtest del ITPA Nivel Representativo: Auditivo-Vocal

\begin{tabular}{|c|c|c|c|c|}
\hline VARIABLES & SUBTEST & MANOVA & $t / F$ & $\bar{x}$ \\
\hline Provincia & $\begin{array}{l}\text { Asociación } \\
\text { Auditiva }\end{array}$ & $\begin{array}{c}\lambda=0,802 \\
F=16,311^{* * *} \\
\eta^{2} \text { parcial }=0,198\end{array}$ & $t=-6,301^{* * * *}$ & $\mathrm{~T}(29,58), \mathrm{LP}(31,13)$ \\
\hline \multirow[t]{2}{*}{ Etapa } & $\begin{array}{l}\text { Comprensión } \\
\text { Auditiva }\end{array}$ & $\begin{array}{c}\lambda=0,906 \\
F=3,318^{* *}\end{array}$ & $t=3,782^{*}$ & $\begin{array}{c}\mathrm{P}(25,53), \mathrm{S}(27,61) \\
\mathrm{B} / \mathrm{FP}(37,60)\end{array}$ \\
\hline & $\begin{array}{l}\text { Expresión } \\
\text { Verbal }\end{array}$ & $\eta^{2}$ parcial $=0,048$ & $t=5,311^{* * *}$ & $\begin{array}{c}\mathrm{P}(29,59), \mathrm{S}(26,39) \\
\mathrm{B} / \mathrm{FP}(40,80)\end{array}$ \\
\hline \multirow{2}{*}{ Adaptación } & $\begin{array}{c}\text { Comprensión } \\
\text { Auditiva } \\
\end{array}$ & \multirow{2}{*}{$\begin{array}{c}\lambda=0,925 \\
F=5,351^{* * * *} \\
\eta^{2} \text { parcial }=0,075\end{array}$} & $t=-4,041^{* * * *}$ & $\begin{array}{l}\text { ACI }(30,67), \\
\text { nACI }(38,47)\end{array}$ \\
\hline & $\begin{array}{c}\text { Asociación } \\
\text { Auditiva }\end{array}$ & & $t=-0,067^{*}$ & $\begin{array}{l}\text { ACI }(29,10), \\
\text { nACI }(29,02)\end{array}$ \\
\hline Recursos & $\begin{array}{l}\text { Asociación } \\
\text { Auditiva }\end{array}$ & $\begin{array}{c}\lambda=0,890 \\
F=2,588^{*} \\
\eta^{2} \text { parcial }=0,038\end{array}$ & $F=6,368^{* * * *}$ & $\begin{array}{l}\mathrm{AL}(35,24), \mathrm{ELSE} \\
(35,67), \mathrm{AL} / \mathrm{ELSE} \\
(25,55), \mathrm{SA}(39,14)\end{array}$ \\
\hline $\begin{array}{l}\text { Horas de } \\
\text { logopedia }\end{array}$ & $\begin{array}{l}\text { Asociación } \\
\text { Auditiva }\end{array}$ & $\begin{array}{c}\lambda=0,880 \\
F=2,137^{*} \\
\eta^{2} \text { parcial }=0,042\end{array}$ & $F=3,640^{* *}$ & $\begin{array}{c}\mathrm{N}(40,94), 1 \mathrm{~h}(35,35), \\
2 \mathrm{~h}(36,71), 3 \mathrm{~h} \\
(31,85), 4 \mathrm{~h}(27,22)\end{array}$ \\
\hline \multirow{2}{*}{$\begin{array}{l}\text { Años experiencia } \\
\text { profesor tutor }\end{array}$} & $\begin{array}{l}\text { Comprensión } \\
\text { Auditiva }\end{array}$ & \multirow{2}{*}{$\begin{array}{c}\lambda=0,901 \\
F=2,318^{*} \\
\eta^{2} \text { parcial }=0,034\end{array}$} & $F=4,377^{* *}$ & $\begin{array}{c}<3(28,01), 4-6 \\
(30,17), 7-9(19,75) \\
>10(22,18)\end{array}$ \\
\hline & $\begin{array}{l}\text { Asociación } \\
\text { Auditiva }\end{array}$ & & $F=4,621^{* *}$ & $\begin{array}{c}<3(30,10), 4-6 \\
(42,13), 7-9 \\
(40,44),>10(33,83) \\
\end{array}$ \\
\hline $\begin{array}{l}\text { Años experiencia } \\
\text { profesor } \\
\text { especialista }\end{array}$ & $\begin{array}{l}\text { Asociación } \\
\text { Auditiva }\end{array}$ & $\begin{array}{c}\lambda=0,908 \\
F=2,152^{*} \\
\eta^{2} \text { parcial }=0,032\end{array}$ & $F=3,594^{*}$ & $\begin{array}{c}<3(35,14), 4-6 \\
(40,86), 7-9 \\
(39,88),>10(32,86)\end{array}$ \\
\hline \multirow{2}{*}{$\begin{array}{l}\text { Estudios de la } \\
\text { madre }\end{array}$} & $\begin{array}{l}\text { Comprensión } \\
\text { Auditiva }\end{array}$ & $\begin{array}{c}\lambda=0,825 \\
F=3,244^{* * *} \\
\eta^{2} \text { parcial }=0,062\end{array}$ & $F=4,046^{* *}$ & $\begin{array}{c}\mathrm{Se}(30,83), \operatorname{Pr}(21,65), \\
\mathrm{Ba}(27,33), \mathrm{Su} \\
(30,25), \mathrm{Fp}(30,80)\end{array}$ \\
\hline & $\begin{array}{l}\text { Expresión } \\
\text { Verbal }\end{array}$ & $\begin{array}{c}\lambda=0,858 \\
F=2,564^{* *} \\
\eta^{2} \text { parcial }=0,050\end{array}$ & $F=3,367^{*}$ & $\begin{array}{c}\mathrm{Se}(26,78), \operatorname{Pr}(25,25), \\
\mathrm{Ba}(29,53), \mathrm{Su} \\
(32,95), \mathrm{Fp}(35,70)\end{array}$ \\
\hline
\end{tabular}

Nota: ${ }^{*} p<0,05 ;{ }^{* *} p<0,010 ;{ }^{* * *} p<0,001$. Provincia: Tenerife (TF), Las Palmas de GC (LP). Etapa (educativa): Primaria (P), Secundaria(S), Bachillerato/FP (B). Adaptación: Adaptación curricular individualizada (ACI), no ACI (nACI). Recursos: Profesor Especialista de Audición y Lenguaje (AL), Especialista en Lengua de Signos Española (ELSE), Ambos (AL/ELSE), Sin apoyos (SA). Horas de logopedia: Ninguna (N), 1h, 2h, 3h, 4h. Años de experiencia profesor tutor o del profesor especialista en audición y lenguaje: Menos de 3 años $(<3)$, entre 4-6 años, entre 7-9 años, más de 10 años (< 10). Estudios de la madre: Sin estudios (Se), Estudios Primarios (Pr), Bachillerato (Ba), Estudios Superiores (Su), Formación Profesional (Fp).

Fuente: Elaboración propia. 
Se destaca también que aquellos que reciben dos horas de logopedia de manera extraescolar alcanzan mejores resultados en los subtests Integración Gramatical $(\bar{x}=$ 24,22) e Integración Visual $(\bar{x}=40,78)$. Resulta llamativo también que los Años de experiencia del profesor tutor (en torno a los 4-6 años) suponen una variable socioeducativa que marca diferencias en los resultados obtenidos por los niños y adolescentes en los subtests Integración Gramatical e Integración Visual.

El tipo de comunicación que se desarrolla en la familia (signos, oral o bilingüe) provoca una media más elevada en Memoria Visomotora para aquellos niños y adolescentes que emplean Signos $(\bar{x}=40,70)$. Sin embargo, en Integración Visual, los mejores resultados los obtienen niños y adolescentes bilingües $(\bar{x}=33,06)$ obteniéndose en ambos casos diferencias estadísticamente significativas $[F(3,617), p<0,001, \lambda$ de Wilks $=0,867$ y eta parcial cuadrado $=0,069]$.

El cuadro 3 refleja cómo las medias fueron más elevadas y con diferencias significativas para la provincia de Las Palmas de Gran Canaria en todos los niveles y subtests del ITPA $[F(16,311), p<0,001, \lambda$ de Wilks $=0,802$ y eta parcial cuadrado $=0,198]$ para el Nivel Representativo Auditivo-Vocal (subtest Asociación Auditiva). El valor de eta parcial cuadrado para la variable Etapa Educativa de los niños y adolescentes proporcionó una explicación del modelo del $48 \%$ de los casos, manifestando, tanto para los subtests Comprensión Auditiva como Expresión Verbal, valores significativos superiores en el caso de los niños y adolescentes que cursaban etapas educativas superiores (Bachillerato y Formación Profesional).

Los niños y adolescentes que tenían Adaptaciones Curriculares Individualizadas (ACIS) (valor explicativo del modelo eta parcial cuadrado del 75\%) obtuvieron valores medios superiores en los subtests del Nivel Representativo Auditivo-Vocal (Comprensión Auditiva y Expresión Verbal) frente a aquellos que no tenían ACIS $(\lambda=0,925, F=5,351$, $p<0,001)$. Contar con Recursos profesionales (Profesores Especialistas en Lengua de Signos Española, ELSE), supuso la obtención de mejores resultados en los subtests del ITPA vinculados a la subescala de Asociación Auditiva, como reflejan los valores $[F$ $(2,588), p<0,05, \lambda$ de Wilks $=0,890$ y eta parcial cuadrado $=0,038]$.

El cuadro 4 refleja cómo las medias fueron más elevadas y con diferencias significativas para aquellos niños y adolescentes con Calificaciones más elevadas en el subtest Expresión Motora del ITPA en el Nivel Representativo Viso-Motor $[F(1,878), p<0,05, \lambda$ de Wilks $=0,893$ y eta parcial cuadrado $=0,037]$ donde los niños y adolescentes que tienen Sobresaliente en sus calificaciones como promedio del curso anterior alcanzan también medias superiores en el citado subtest.

De otra parte, los Años de experiencia, tanto del profesor tutor como del profesor especialista se relacionan con los resultados obtenidos por los niños y adolescentes en los subtests Comprensión Visual y Expresión motora, en el primer caso y con Asociación Visual en el segundo. En todos los casos, la media superior la obtiene el profesorado que tiene entre 4 y 6 años de experiencia docente. Los estudios del padre se relacionan con los resultados obtenidos en el subtest Comprensión Visual $[F(3,244), p<0,001, \lambda$ de Wilks $=0,825$, eta parcial cuadrado $=0,062]$ siendo los Estudios superiores los que obtienen la media más elevada $(\bar{x}=32,53)$. 
Cuadro 4. Resultados del MANOVA por variables socioeducativas de niños y adolescentes con IC o AUD en el Subtest del ITPA Nivel Representativo: Viso-Motor

\begin{tabular}{|c|c|c|c|c|}
\hline VARIABLES & SUBTEST & MANOVA & $t / F$ & $\bar{x}$ \\
\hline \multirow{2}{*}{ Provincia } & $\begin{array}{c}\text { Comprensión } \\
\text { Visual }\end{array}$ & \multirow{2}{*}{$\begin{array}{c}\lambda=0,923 \\
F=5,513^{* * *} \\
\eta^{2} \text { parcial }=0,077\end{array}$} & $t=-3,915^{* * * *}$ & $\begin{array}{l}\mathrm{T}(32,24), \\
\mathrm{LP}(37,82)\end{array}$ \\
\hline & $\begin{array}{c}\text { Expresión } \\
\text { Motora }\end{array}$ & & $t=-2,807^{* *}$ & $\begin{array}{c}\mathrm{T}(29,59), \\
\operatorname{LP}(38,76)\end{array}$ \\
\hline \multirow[b]{2}{*}{ Recursos } & $\begin{array}{c}\text { Comprensión } \\
\text { Visual }\end{array}$ & \multirow{2}{*}{$\begin{array}{c}\lambda=0,901 \\
F=2,332^{*} \\
\eta^{2} \text { parcial }=0,034\end{array}$} & $F=4,573^{* *}$ & $\begin{array}{c}\text { AL }(34,99), \text { SA }(37,36) \\
\text { ELSE }(36,25), \\
\text { AL/ELSE }(28,83) \\
\end{array}$ \\
\hline & $\begin{array}{l}\text { Expresión } \\
\text { Motora }\end{array}$ & & $F=5,197^{* *}$ & $\begin{array}{c}\mathrm{AL}(32,38), \mathrm{SA}(33,80) \\
\mathrm{ELSE}(30,92), \\
\mathrm{L} / \mathrm{ELSE}(24,55)\end{array}$ \\
\hline Calificación & $\begin{array}{l}\text { Expresión } \\
\text { Motora }\end{array}$ & $\begin{array}{c}\lambda=0,893 \\
F=1,878^{*} \\
\eta^{2} \text { parcial }=0,037\end{array}$ & $F=4,822^{* * * *}$ & $\begin{array}{c}\mathrm{I}(30,31), \mathrm{S}(29,06) \\
\mathrm{B}(30,69), \mathrm{N}(37,46) \\
\text { So }(37,78)\end{array}$ \\
\hline \multirow{2}{*}{$\begin{array}{c}\text { Años experiencia } \\
\text { profesor tutor }\end{array}$} & $\begin{array}{c}\text { Comprensión } \\
\text { Visual }\end{array}$ & \multirow{2}{*}{$\begin{array}{c}\lambda=0,852 \\
F=3,482^{*} \\
\eta^{2} \text { parcial }=0,052\end{array}$} & $F=8,207^{* * *}$ & $\begin{array}{c}<3(26,90), 4-6 \\
(38,56), 7-9(37,69) \\
>10(35,61)\end{array}$ \\
\hline & $\begin{array}{l}\text { Expresión } \\
\text { Motora }\end{array}$ & & $F=4,522^{*}$ & $\begin{array}{c}<3(26,03), 4-6 \\
(37,69), 7-9(32,78) \\
>10(31,86)\end{array}$ \\
\hline $\begin{array}{c}\text { Años experiencia } \\
\text { profesor } \\
\text { especialista }\end{array}$ & $\begin{array}{l}\text { Asociación } \\
\text { Visual }\end{array}$ & $\begin{array}{c}\lambda=0,896 \\
F=2,444^{*} \\
\eta^{2} \text { parcial }=0,036\end{array}$ & $F=4,572^{*}$ & $\begin{array}{c}<3(32,71), 4-6 \\
(44,86), 7-9(34,98) \\
>10(35,41)\end{array}$ \\
\hline $\begin{array}{l}\text { Estudios del } \\
\text { padre }\end{array}$ & $\begin{array}{c}\text { Comprensión } \\
\text { Visual }\end{array}$ & $\begin{array}{c}\lambda=0,825 \\
F=3,244^{* * *} \\
\eta^{2} \text { parcial }=0,062\end{array}$ & $\mathrm{~F}=4,817^{* * * *}$ & $\begin{array}{c}\mathrm{Se}(29,41), \operatorname{Pr}(22,62), \\
\mathrm{Ba}(24,00), \mathrm{Su}(32,53), \\
\operatorname{Fp}(30,58)\end{array}$ \\
\hline
\end{tabular}

Nota: ${ }^{*} p<0,05 ;{ }^{* *} p<0,010 ;{ }^{* * *} p<0,001$.

Fuente: Elaboración propia.

\subsection{Relación entre las subescalas de SDQ y los niveles del ITPA en niños y adolescentes}

Como se indica en el cuadro 5, las subescalas de ITPA se correlacionaron significativamente entre sí, por ejemplo, Comprensión Auditiva con Expresión Verbal $(r$ $=0,616, p<0,001)$, Comprensión Auditiva con Memoria Auditiva $(r=0,632, p<0,001)$, y Comprensión Auditiva con Integración Gramatical $(r=0,581, p<0,001)$. Estas correlaciones mostraron una asociación entre dos niveles de organización de complejidad creciente. Las correlaciones entre las subescalas de ITPA y las de SDQ son muy bajas o no hay correlación, lo que refleja que cada prueba es independiente y mide aspectos claramente diferentes.

\subsection{Modelo de predicción de puntuaciones de ITPA en niños y adolescentes}

Los resultados del análisis de regresión se muestran en el cuadro 6, donde algunas subescalas de ITPA explican el 42,6\% de la varianza de la puntuación total de SDQ de los niños y adolescentes. El Nivel Automático Auditivo-Vocal tiene un peso importante en la puntuación de las fortalezas y debilidades de la puntuación total de SDQ de los niños y adolescentes, específicamente, las escalas Memoria Secuencial Auditiva, y con un valor significativo aún mayor Integración Gramatical $(t=3,781, p<0,001)$. 
Cuadro 5. Correlaciones entre las subescalas de SDQ y las puntuaciones de niveles del ITPA en niños y adolescentes con IC o AUD

\begin{tabular}{|c|c|c|c|c|c|c|c|c|c|c|c|c|c|c|c|}
\hline & S1 & SD & S3 & S4 & S5 & I1 & I2 & I3 & I4 & I5 & I6 & I7 & I8 & I9 & I10 \\
\hline S1 & 1,000 &, $283^{* * * *}$ & $-0,232^{* * * *}$ & $-0,207^{* *}$ & $0,151^{*}$ & $0,137^{*}$ & 0,101 & 0,047 & $-0,044$ & $-0,020$ & 0,047 & 0,023 & $0,149^{*}$ & $0,169^{*}$ & 0,129 \\
\hline $\mathrm{S} 2$ & $0,283^{* * *}$ & 1,000 & $0,226^{* * * *}$ & $0,288^{* * * *}$ & $-0,334^{* * * *}$ & $-0,012$ & $-0,063$ & 0,081 & 0,003 & 0,054 & $-0,050$ & 0,082 & 0,004 & $-0,130$ & 0,047 \\
\hline S3 & $-0,232^{* *}$ & $0,226^{* * *}$ & 1,000 & $0,473^{* * * *}$ & $-0,327^{* * * *}$ & $-0,003$ & $-0,149^{*}$ & 0,117 & $-0,032$ & 0,029 & $-0,078$ & 0,060 & $-0,079$ & $-0,130$ & $-0,103$ \\
\hline S4 & $-0,207^{* *}$ & $0,288^{* * * *}$ & $0,473^{* * * *}$ & 1,000 & $-0,386^{* * * *}$ & $-0,051$ & $-0,150^{*}$ & 0,105 & $-0,064$ & 0,006 & $-0,093$ & $-0,012$ & $-0,103$ & $-0,103$ & $-0,136^{*}$ \\
\hline S5 & $0,151^{*}$ & $-0,334^{* * *}$ & $-0,327^{* * * *}$ & $-0,386^{* * *}$ & 1,000 & 0,031 & 0,130 & -0,099 & 0,002 & $-0,048$ & 0,066 & $-0,081$ & $0,141^{*}$ & 0,129 & 0,055 \\
\hline I 1 & $0,137^{*}$ & $-0,012$ & $-0,003$ & $-0,051$ & 0,031 & 1,000 & $0,230^{* * * *}$ & $0,616^{* * * *}$ & $-0,033$ & $0,268^{* * * *}$ & 0,052 & $0,632^{* * *}$ & $0,581^{* * *}$ & $0,309^{* * * *}$ & 0,003 \\
\hline I 2 & 0,101 & $-0,063$ & $-0,149^{*}$ & $-0,150^{*}$ & 0,130 & $0,230^{* * * *}$ & 1,000 & 0,023 & $0,305^{* * * *}$ & 0,114 & $0,635^{* * * *}$ & $0,188^{* *}$ & $0,576^{* * *}$ & 0,055 & $0,277^{* * * *}$ \\
\hline I3 & 0,047 & 0,081 & 0,117 & 0,105 & $-0,099$ & $0,616^{* * * *}$ & 0,023 & 1,000 & $-0,133$ & $0,302^{* * * *}$ & 0,019 & $0,486^{* * * *}$ & $0,462^{* * *}$ & $0,324^{* * * *}$ & $-0,042$ \\
\hline I 4 & $-0,044$ & 0,003 & $-0,032$ & $-0,064$ & 0,002 & $-0,033$ & $0,305^{* * *}$ & $-0,133$ & 1,000 & $0,241^{* * *}$ & $0,467^{* * * *}$ & $-0,102$ & $0,218^{* *}$ & $-0,064$ & 0,097 \\
\hline $\mathrm{I} 5$ & $-0,020$ & 0,054 & 0,029 & 0,006 & $-0,048$ & $0,268^{* * * *}$ & 0,114 & $0,302^{* * * *}$ & $0,241^{* * * *}$ & 1,000 & 0,068 & $0,242^{* *}$ & $0,300^{* * *}$ & $-0,028$ & $-0,060$ \\
\hline I6 & 0,047 & $-0,050$ & $-0,078$ & $-0,093$ & 0,066 & 0,052 & $0,635^{* * *}$ & 0,019 & $0,467^{* * * *}$ & 0,068 & 1,000 & 0,035 & $0,457^{* * * *}$ & $0,149^{*}$ & $0,218^{* *}$ \\
\hline I7 & 0,023 & 0,082 & 0,060 & $-0,012$ & $-0,081$ & $0,632^{* * * *}$ & $0,188^{* * *}$ & $0,486^{* * * *}$ & $-0,102$ & $0,242^{* * *}$ & 0,035 & 1,000 & $0,435^{* * * *}$ & $0,215^{* *}$ & $-0,010$ \\
\hline I8 & $0,149^{*}$ & 0,004 & $-0,079$ & $-0,103$ & $0,141^{*}$ & $0,581^{* * * *}$ & $0,576^{* * *}$ & $0,462^{* * * *}$ & $0,218^{* *}$ & $0,300^{* * * *}$ & $0,457^{* * * *}$ & $0,435^{* * * *}$ & 1,000 & $0,208^{* *}$ & $0,250^{* * * *}$ \\
\hline I9 & $0,169^{*}$ & $-0,130$ & $-0,130$ & $-0,103$ & 0,129 & $0,309^{* * * *}$ & 0,055 & $0,324^{* * * *}$ & $-0,064$ & $-0,028$ & $0,149^{*}$ & $0,215^{* *}$ & $0,208^{* *}$ & 1,000 & $-0,127$ \\
\hline I10 & 0,129 & 0,047 & $-0,103$ & $-0,136^{*}$ & 0,055 & $0,003^{* *}$ & $0,277^{* * *}$ & $-0,042$ & 0,097 & $-0,060$ & $0,218^{* *}$ & $-0,010$ & $0,250^{* * *}$ & $-0,127$ & 1,000 \\
\hline
\end{tabular}

Nota: ${ }^{*} p<0,05 ;{ }^{* *} p<0,010 ;{ }^{* * *} p<0,001$. SDQ Subescalas: S1: Problemas emocionales; S2: Problemas conductuales; S3: Hiperactividad; S4: Problemas con los compañeros; S5: Conducta prosocial. ITPA Subescalas: I 1: Nivel Representativo: Auditivo-Vocal Comprensión Auditiva; I2: Nivel Representativo: Auditivo-Vocal Asociación Auditiva;

I3: Nivel Representativo: Auditivo-Vocal Expresión Verbal; I4: Nivel Representativo Viso-Motor Comprensión Visual; I5: Nivel Representativo Viso-Motor Asociación Visual; I6: Nivel Representativo Viso-Motor Expresión Motora; I7: Nivel Automático Auditivo-Vocal Memoria Secuencial Auditiva; I8: Nivel Automático AuditivoVocal Integración Gramatical; I9: Nivel Automático Auditivo-Motor Memoria Secuencial Visomotora; I10: Nivel Automático Auditivo-Vocal (test complementario) Integración Auditiva.

Fuente: Elaboración propia. 
En el Nivel Representativo Auditivo-Vocal la escala Expresión Verbal obtiene un destacado nivel de significación $(t=-2,846, p<0,010)$ y el Nivel Representativo VisoMotor en la escala de Comprensión Visual también tiene un destacado peso significativo $(t=-2,683, p<0,010)$.

Cuadro 6. Análisis de regresión de la puntuación total de SDQ de los niños y adolescentes con IC o AUD como predictor del ITPA

\begin{tabular}{|c|c|c|c|c|c|c|}
\hline $\mathbf{R}$ & $\mathbf{R}^{2}$ & $\mathbf{F}$ & $\begin{array}{r}\text { VARIANZA } \\
\text { EXPLICADA }\end{array}$ & SUBTEST ITPA & $\beta$ & $t$ \\
\hline \multirow{5}{*}{0,426} & \multirow{5}{*}{0,181} & \multirow{5}{*}{$8,594^{* * * *}$} & \multirow{5}{*}{$42,6 \%$} & $\begin{array}{l}\text { Nivel Automático Auditivo- } \\
\text { Motor Memoria Secuencial } \\
\text { Visomotora }\end{array}$ & 0,022 & $4,502^{* * * *}$ \\
\hline & & & & $\begin{array}{l}\text { Nivel Automático Auditivo- } \\
\text { Vocal Memoria Secuencial } \\
\text { Auditiva }\end{array}$ & $-0,014$ & $-2,934^{* * *}$ \\
\hline & & & & $\begin{array}{l}\text { Nivel Automático Auditivo- } \\
\text { Vocal Integración } \\
\text { Gramatical }\end{array}$ & 0,015 & $3,781^{* * *}$ \\
\hline & & & & $\begin{array}{l}\text { Nivel Representativo: } \\
\text { Auditivo-Vocal Expresión } \\
\text { Verbal }\end{array}$ & $-0,014$ & $-2,846^{* *}$ \\
\hline & & & & $\begin{array}{l}\text { Nivel Representativo Viso- } \\
\text { Motor Comprensión Visual }\end{array}$ & $-0,014$ & $-2,683^{* *}$ \\
\hline
\end{tabular}

Nota: ${ }^{*} p<0,05 ;{ }^{* *} p<0,010 ;{ }^{* * *} p<0,001$.

Fuente: Elaboración propia.

El valor $F$ obtenido es de 8,594 , cuya probabilidad asociada de acuerdo con las expectativas de la hipótesis nula es inferior a 0,001 , lo que lleva a los autores a rechazar esta hipótesis y suponer que hay un efecto real de estas variables en la puntuación total de SDQ de niños y adolescentes. Por lo tanto, se puede afirmar que la puntuación total de SDQ está claramente influenciada por los hábitos integrados, como la memoria y el aprendizaje remoto que producen una cadena automática de respuestas, así como las modalidades visuales o auditivas que constituyen la entrada del canal de comunicación, resaltando la influencia más alta del Nivel Automático Auditivo-Motor en la Memoria Secuencial Visomotora $(t=4,502, p<0,001)$.

\section{Discusión y conclusiones}

Los hallazgos han mostrado, conforme se había hipotetizado para la meta 1, que las percepciones sobre las fortalezas y debilidades de los niños y adolescentes con IC o AUD, medidas por las subescalas de SDQ, fueron distintas en los grupos estudiados: niños y niñas, familias y profesores especialistas y tutores. De acuerdo con las autopercepciones de los niños y adolescentes, las medias de tres subescalas de SDQ fueron distintas: Problemas de conducta, Hiperactividad y Problemas con compañeros, siendo los puntajes menos favorables para aquellos con dificultades auditivas que emplean IC. Estas diferencias en la sintomatología emocional de los niños y adolescentes con IC o AUD fue más evidente en los puntajes totales de sus familias. Al igual que los estudios de Huber y Kipman (2011) en Austria o Anmyr y otros (2012) en Suecia, las familias de niños y adolescentes con IC o AUD analizados percibieron que estos fueron distintos entre sí en 
cuatro subescalas de SDQ: Problemas de conducta, Hiperactividad, Problemas con compañeros y Prosocial.

El presente estudio mantuvo una orientación distinta al descubrimiento de Bat-Chava, Martín y Kosciw (2005) que aludía al alineamiento en las percepciones de los padres y maestros sobre las destrezas de comunicación de los niños y adolescentes con IC. El profesorado tutor percibió, por su parte, que los niños y adolescentes con IC o AUD fueron diferentes en dos subescalas de SDQ: Problemas de conducta e Hiperactividad, siendo más elevadas las puntuaciones en esta última escala para aquellos niños y adolescentes que emplean IC. Asimismo, los hallazgos han hecho patente, conforme se había hipotetizado en la meta 2 , que los niños y adolescentes con IC o AUD puntuaban de manera diferente según las variables socioeducativas propuestas. Este estudio ha aportado la novedad de analizar la aplicación del ITPA a niños y adolescentes con IC o AUD, que no se había hecho en ninguno de los 68 estudios revisados por Kirk y Elkins (1974).

Los niños y adolescentes con dificultades auditivas percibieron de manera diferente la habilidad para reproducir de memoria secuencias de figuras no significativas después de ver la secuencia durante un breve periodo de tiempo, obteniendo mejor resultado los niños y adolescentes que empleaban AUD, obteniéndose diferencias en función del lugar se residencia familiar, igual que obtuvo Fernández-Batanero (2015) en su estudio sobre la dinámica familiar. Analizada la Etapa educativa, los niños y adolescentes de Bachillerato/FP fueron superiores que los de Educación Primaria y Educación Secundaria en la habilidad para obtener significado a partir de material presentado oralmente, así como en la fluidez verbal de niños y adolescentes, medida a partir del número de conceptos expresados verbalmente.

En cuanto a la realización de un currículo adaptado, los niños y adolescentes con ACI fueron superiores que quienes no tenían ACI en la habilidad para obtener significado a partir del material presentado oralmente y en la fluidez verbal, mientras que los que no tenían ACI lo fueron en la habilidad para relacionar conceptos presentados visualmente y en la puntuación global del ITPA. Como investigó Hintermair (2013), la inclusión de un programa formativo de funciones ejecutivas contribuiría a mejorar la comunicación de estudiantes con dificultades auditivas. Desde el punto de vista de la evaluación del rendimiento del curso previamente realizado, los niños y adolescentes con dificultades auditivas obtuvieron resultados diferentes en el ITPA, según las calificaciones del curso anterior. Las medias de los niños y adolescentes que obtuvieron la calificación de Sobresaliente reflejaron medias superiores a los de las restantes calificaciones en distintas habilidades como fueron: expresar significados mediante gestos manuales; identificar animales u objetos conocidos a partir de una representación incompleta de los mismos, en un contex to relativamente complejo, y reproducir secuencias de palabras, números, letras, o símbolos, presentados visualmente.

Las sesiones extraescolares de logopedia tenían como objeto intervenir en los problemas de comunicación. Los niños y adolescentes analizados se diferenciaron entre sí con valores medios superiores cuando asistieron dos horas semanales a clases de logopedia, en la habilidad para identificar animales u objetos conocidos a partir de una representación incompleta de los mismos, en un contex to relativamente complejo. La experiencia docente del profesorado tutor (entre 4 y 6 años) identificó significativas diferencias entre los niños 
y adolescentes en dos habilidades del ITPA correspondientes al Nivel Automático: Auditivo-Vocal, la Integración Gramatical y la Integración Visual. Además, el profesorado tutor que tenía entre 4-6 años de experiencia docente diferenciaron a los niños y adolescentes con medias más elevadas en las siguientes habilidades: Comprensión Auditiva, Asociación Auditiva, Comprensión Visual, Expresión Motora, Integración Gramatical e Integración Visual. Mientras que la otra habilidad que provocó diferencias entre los niños y adolescentes (relacionar conceptos presentados visualmente), se vinculó con la experiencia de 4-6 años del profesorado especialista.

Estudiado el nivel formativo de la familia, los padres con estudios superiores manifestaron diferencias entre los niños y adolescentes con dificultades auditivas en dos destrezas: adquirir significado de símbolos visuales, eligiendo, a partir de un conjunto de dibujos, el que fuera semejante al dibujo-estímulo, y retener a corto plazo toda la información auditiva que los niños y adolescentes recibían del entorno. Los estudios superiores o de formación profesional de la madre expresaron una diferencia en los niños y adolescentes con dificultades auditivas en la habilidad para adquirir significado a partir de material presentado oralmente. Además, cuando las madres estaban desempleadas, los niños y adolescentes con IC o AUD fueron distintos en el subtest que evaluaba la fluidez verbal del mismo, medida a partir del número de conceptos expresados verbalmente. De otra parte, la comunicación por medio de la lengua de signos evidenció que los niños y adolescentes con dificultades auditivas fueron distintos en el subtest Memoria Visual, mientras que el uso de la comunicación oral provocó que los niños y adolescentes fueran distintos en la habilidad para identificar animales u objetos conocidos a partir de una representación incompleta de los mismos, en un contexto relativamente complejo.

Las correlaciones entre las puntuaciones de ITPA y SDQ (meta 3) mostraron una relación baja en este estudio. En cualquier caso, se ha observado anteriormente que el ITPA mantiene correlaciones bajas con otras pruebas (Kirk y Elkins, 1974). En general, tres subtests auditivos de ITPA están relacionadas entre sí: Comprensión Auditiva con Expresión Verbal, Comprensión Auditiva con Memoria Auditiva, y Comprensión Auditiva con Integración Gramatical. Finalmente, se obtuvo (meta 4), mediante un análisis de regresión, que algunas escalas de ITPA explicaron $42.6 \%$ de la varianza de la puntuación total de SDQ de los niños y adolescentes con dificultades auditivas del presente estudio. Destacaron por su peso en el valor t y la significatividad los subtests Memoria Secuencial Visomotora, Integración Gramatical, Memoria Secuencial Auditiva, Expresión Verbal y la Comprensión Visual.

Como conclusiones generales, los resultados revelaron la necesidad de armonizar intervenciones psicoeducativas para los grupos de sujetos analizados que puntuaron la Escala de Problemas de conducta del SDQ como la cuestión emocional y social de mayor dificultad y que mejor distinguía a los niños y adolescentes con IC de los que usaban AUD.

También ha quedado demostrado que las variables socioeducativas planteadas en el estudio están relacionadas con los resultados obtenidos por los niños y adolescentes con IC o AUD en el test ITPA. Las subescalas del SDQ y subtests de ITPA no se correlacionan entre si expresando ambos idoneidad y expectativas de que miden de manera independiente lo que pretenden medir. Finalmente, la puntuación total de niños y adolescentes con dificultades auditivas que obtienen en SDQ es un buen predictor de los valores obtenidos en los subtests de ITPA, especialmente en aquellos subtests vinculados 
a la memoria secuencial visomotora y secuencial auditiva, así como para la integración gramatical, la expresión verbal y la compresión visual.

Este estudio presenta ciertas limitaciones. No se ha estudiado las interacciones entre los problemas de los niños con IC o AUD y los problemas de sus pares (Huber y Kipman, 2011) o de estudiantes con dificultades auditivas y otros déficits asociados mediante instrumentos complementarios (Torras-Mañá et al., 2016).

Se sugiere proponer programas psicoeducativos que promuevan el desarrollo emocional y habilidades sintácticas, de lectura, vocabulario y estrategias semánticas para niños y adolescentes que emplean IC o AUD (Domínguez et al., 2016). En particular, programas implantados por maestros especialistas en audición y lenguaje que participan con los maestros tutores en la mejora de problemas de conducta y de comprensión del significado de materiales curriculares presentados oralmente en la enseñanza. También conviene plantear estrategias específicas en los subtests del ITPA Memoria Secuencial Visomotora, Integración Gramatical, Memoria Secuencial Auditiva, Expresión Verbal y la Comprensión Visual puesto que se ha demostrado la influencia específica que la puntuación total de SDQ tiene sobre los mismos.

Se propone establecer grupos de padres y profesores para poner en común percepciones y opiniones relacionadas con el equilibrio emocional de los niños y adolescentes con dificultades auditivas, a fin de armonizar criterios cara a mantener en el hogar el necesario apoyo emocional de los hijos y en la escuela el desarrollo de estrategias didácticas específicas orientadas en la misma dirección.

\section{Referencias}

Anmyr, L., Larsson, K., Olsson, M. y Freijd, A. (2012). Strengths and difficulties in children with cochlear implants. Comparing self-reports with reports from parents and teachers. International Journal of Pediatric Otorhinolaryngology, 76, 1107-1112. https://doi.org/10.1016/j.ijporl.2012.04.009

Bat-Chava, Y., Martín, D. y Kosciw, J. G. (2005). Longitudinal improvements in communication and socialization of deaf children with cochlear implants and hearing aids: Evidence from parental reports. Journal of Child Psychology and Psychiatry, 46(12), 1287-1296. https://doi.org/10.1111/j.1469-7610.2005.01426.x

Bateman, B. (1965). The Illinois test of psycholinguistic abilities in current research. Summaries of studies. Champaign, IL: Inst. of Research for Exceptional Children.

Calderón, R. y Greenberg, M. T. (2003). Social and emotional development of deaf children. En M. Marcharck y P. E. Spencer (Eds.), Deaf studies, language and education (pp. 434-448). Nueva York, NY: Oxford University Press.

Dammeyer, J. (2010). Psychosocial development in a Danish population of children with cochlear implants and deaf and hard-of-hearing children. Journal of Deaf Studies and Deaf Education, 15, 50-58. https://doi.org/10.1093/deafed/enpo24

Domínguez, A. B., Carrillo, M. S., González, V. y Alegria, J. (2016). How do deaf children with and without cochlear implants manage to read sentences: The key word strategy. Journal of Deaf Studies and Deaf Education, 21(3), 280-292. https://doi.org/10.1093/deafed/enw026 
Fernández-Batanero, J. M. (2015). Dinámica familiar e implante coclear: Estudio de casos. Infancia y Aprendizaje, 38(1), 30-41. https://doi.org/10.1080/02 103702.2014.996404

Girbau, D. (2016). The non-word repetition task as a clinical marker of specific language impairment in Spanish-speaking children. First Language, 36(1), 30-49. https://doi.org/10.1177\%2Fo142723715626069

Goodman, R. (2001). Psychometric properties of the strengths and difficulties questionnaire. Journal of the American Academy of Child \& Adolescent Psychiatry, 40, 1337-1345. https://doi.org/10.1097/00004583-200111000-00015

Goodman, A. y Goodman, R. (2009). Strengths and difficulties questionnaire as a dimensional measure of child mental health. Journal of the American Academy of Child \& Adolescent Psychiatry, 48(4), 400-403. https://doi.org/10.1097/CHI.obo13e3181985068

Gustafsson, B. M., Proczkowska-Björklund, M. y Gustafsson, P. A. (2017). Emotional and behavioural problems in Swedish preschool children rated by preschool teachers with the strengths and difficulties questionnaire (SDQ). BMC Pediatrics, 17(1), 1-10. https://doi.org/10.1186/s12887-017-0864-2

Hassan, H. E., Eeldin, S. T. K. y Al Kasaby, R. M. (2014). Psycholinguistic abilities in cochlear implant and hearing impaired children. Egyptian Society of Ear, Nose, Throat and Allied Sciences, 15, 29-35. https://doi.org/10.1016/j.ejenta.2013.12.006

Hintermair, M. (2006). Parental resources, parental stress, and socioemotional development of deaf and hard of hearing children. The Journal of Deaf Studies and Deaf Education, 11(4), 493-513. https://doi.org/10.1093/deafed/enlo05

Hintermair, M. (2013). Executive functions and behavioral problems in deaf and hard-of-hearing students at general and special schools. The Journal of Deaf Studies and Deaf Education, 18(3), 344-359. https://doi.org/10.1093/deafed/entoo3

Huber, M. y Kipman, U. (2011). The mental health of deaf adolescents with cochlear implants compared to their hearing peers. International Journal of Audiology, 50, 146-154. https://doi.org/10.3109/14992027.2010.533704

Kirk, S. A. y Elkins, J. (1974). Summaries of research on the revised Illinois test of psycholinguistics. Final report. Tucson, AZ: Deparment of Special Education.

León, M. J., Crisol, E. y Moreno, R. (2018). Las tareas del líder inclusivo en centros educativos de zonas desfavorecidas y favorecidas. REICE. Revista Iberoamericana sobre Calidad, Eficacia y Cambio en Educación, 16(2), 2 1-40. https://doi.org/10.15366/reice2018.16.2.002

Niclasen, J. y Dammeyer, J. (2016). Psychometric properties of the strengths and difficulties questionnaire and mental health problems among children with hearing loss. Journal of Deaf Studies and Deaf Education, 21(2), 129-140. https://doi.org/10.1093/deafed/env067

Organización Mundial de la Salud. (2005). Child and adolescent mental health policies and plans. Recuperado de http://www.who.int/mental_health/policy/Childado_mh_module.pdf

Ortuño-Sierra, J., Fonseca-Pedrero, E., Inchausti, F. y Sastre i Riba, S. (2016). Evaluación de dificultades emocionales y comportamentales en población infanto-juvenil: El cuestionario de capacidades y dificultades (SDQ). Papeles del Psicólogo, 37(1), 14-26.

Ottem, E. (2010). The complementary nature of ITPA and WISC-R. Results for languageimpaired children. Scandinavian Journal of Educational Research, 46(2), 145-160. https://doi.org/10.1080/00313830220142173

Rodríguez-Hernández, P. J., Betancort, M., Ramírez-Santana, G. M., García, R., Sanz-Alvarez, E. J., y De las Cuevas-Castresana, C. (2014). Puntos de corte de la versión española del 
cuestionario de cualidades y dificultades (SDQ). Revista de Psiquiatría Infanto-Juvenil, 3, 2329.

Ruchkin, V., Koposov, R., Vermeiren, R. y Schwab-Stone, M. (2012). The strength and difficulties questionnaire: Russian validation of the teacher version and comparison of teacher and student reports. Journal of Adolescence, 35, 87-96. https://doi.org/10.1016/j.adolescence.2011.06.003

Sharp, C., Croudace, T. J., Goodyer, I. M. y Amtmann, D. (2005). The strength and difficulties questionnaire: Predictive validity of parent and teacher ratings for help-seeking behaviour over one year. Educational and Child Psychology, 22(3), 28-44.

Torras-Mañá, M., Gómez-Morales, A., González-Gimeno, I., Fornieles-Deu, A. y Brun-Gasca, C. (2016). Assessment of cognition and language in the early diagnosis of autism spectrum disorder: Usefulness of the Bayley scales of infant and toddler development. Journal of Intellectual Disability Research, 6O(5), 502-511. https://doi.org/10.1111/jir.12291

Weener, P., Barritt, L. S. y Semmel, M. I. (1967). A critical evaluation of the Illinois test of psycholinguistic abilities. Exceptional Children, 33(6), 373-380. https://doi.org/10.1177/001440296703300602

\section{Breve CV de los autores}

\section{Olga María Alegre}

Doctora en Psicología. Doctora en Ciencias de la Información. Catedrática de Universidad. Departamento de Didáctica e Investigación Educativa de la Universidad de La Laguna. Responsable del Grupo de Investigación de la Comunidad Autónoma de Canarias para la Educación en la Diversidad (GIED). Directora del Centro de Estudios Universitarios para la Educación en la Diversidad. Línea de investigación vinculada la inclusión, calidad, evaluación y desarrollo profesional docente. Posee publicaciones nacionales e internacionales de impacto, lidera proyectos de investigación competitivos y dirige tesis vinculadas al ámbito de la calidad y la inclusión en distintos niveles del sistema educativo. Ha codirigido el International Journal of University Teaching and Faculty Development. ORCID ID: https://orcid.org/O000-0003-0504-0848. Email: oalegre@gmail.com

\section{Luis Miguel Villar}

Doctor en Ciencias de la Educación. Catedrático de Universidad. Departamento de Didáctica y Organización Educativa. Facultad de Educación de la Universidad de Sevilla. Posee publicaciones nacionales e internacionales en el ámbito de la formación del profesorado, calidad, evaluación y desarrollo profesional docente. Ha liderado proyectos de investigación competitivos y dirigido tesis en la línea de investigación vinculada a la enseñanza y formación del profesorado en los distintos niveles del sistema educativo y en diferentes países. Ha codirigido el International Journal of University Teaching and Faculty Development. Presidente de comités de evaluación de la calidad y acreditación de titulaciones y universidades. ORCID ID: https://orcid.org/OOOO-0002-4396-1405. Email: mvillar@us.es 